\title{
Universal Growth Scheme for Quantum Dots with Low Fine-Structure Splitting at Various Emission Wavelengths
}

\author{
Joanna Skiba-Szymanska, ${ }^{1,}$ R. Mark Stevenson, ${ }^{1}$ Christiana Varnava, ${ }^{1,2}$ Martin Felle, ${ }^{1,2}$ Jan Huwer, ${ }^{1}$ Tina Müller, ${ }^{1}$ \\ Anthony J. Bennett, ${ }^{1}$ James P. Lee, ${ }^{1,2}$ Ian Farrer, ${ }^{3,4}$ Andrey B. Krysa ${ }^{4}$ Peter Spencer, ${ }^{3}$ Lucy E. Goff, ${ }^{3}$ \\ David A. Ritchie, ${ }^{3}$ Jon Heffernan, ${ }^{4}$ and Andrew J. Shields ${ }^{1}$ \\ ${ }^{1}$ Toshiba Research Europe Limited, 208 Science Park, Milton Road, \\ Cambridge CB4 OGZ, United Kingdom \\ ${ }^{2}$ Cambridge University Engineering Department, $9 \mathrm{~J} . \mathrm{J}$. Thomson Avenue, \\ Cambridge CB3 OFA, United Kingdom \\ ${ }^{3}$ Cavendish Laboratory, University of Cambridge, J. J. Thomson Avenue, \\ Cambridge CB3 OHE, United Kingdom \\ ${ }^{4}$ EPSRC National Centre for III-V Technologies, Department of Electronic and Electrical Engineering, \\ University of Sheffield, Sheffield S1 3JD, United Kingdom \\ (Received 5 September 2016; revised manuscript received 31 March 2017; published 14 July 2017)
}

\begin{abstract}
Efficient sources of individual pairs of entangled photons are required for quantum networks to operate using fiber-optic infrastructure. Entangled light can be generated by quantum dots (QDs) with naturally small fine-structure splitting (FSS) between exciton eigenstates. Moreover, QDs can be engineered to emit at standard telecom wavelengths. To achieve sufficient signal intensity for applications, QDs have been incorporated into one-dimensional optical microcavities. However, combining these properties in a single device has so far proved elusive. Here, we introduce a growth strategy to realize QDs with small FSS in the conventional telecom band, and within an optical cavity. Our approach employs "droplet-epitaxy" of InAs quantum dots on (001) substrates. We show the scheme improves the symmetry of the dots by $72 \%$. Furthermore, our technique is universal, and produces low FSS QDs by molecular beam epitaxy on GaAs emitting at $\sim 900 \mathrm{~nm}$, and metal-organic vapor-phase epitaxy on InP emitting at $\sim 1550 \mathrm{~nm}$, with mean FSS $4 \times$ smaller than for Stranski-Krastanow QDs.
\end{abstract}

DOI: 10.1103/PhysRevApplied.8.014013

\section{INTRODUCTION}

Entangled photon-pair sources based on the radiative decay of the biexciton state within semiconductor quantum dots [1,2] have many potential advantages, including electrical operation [3], sub-Poissonian statistics, the potential for integration with conventional optoelectronics, and a wide wavelength coverage [4]. Quantum dots (QDs) typically present an energetic fine-structure splitting (FSS) between the exciton eigenstates much larger than the natural linewidth of the transition [5,6], providing "which path" information complicating the observation of entanglement. The FSS originates from asymmetry in the QD wave function caused by variations in crystal strain and composition, and the elongation of the QD shape which itself is a natural consequence of the Stranski-Krastanow (SK) growth technique.

Production of quantum dots with naturally small FSS may be achieved in special cases, by self-assembled growth of InAs quantum dots around $885 \mathrm{~nm}$, where an inversion of the wave-function symmetry is observed [7], or growth on (111) surfaces [8-10], aided by the underlying $C_{3 v}$

\footnotetext{
*To whom correspondence should be addressed. joanna.skiba@crl.toshiba.co.uk
}

crystal symmetry which very often results in dot density preventing the spectral isolation of individual lines [11-13]. Another approach is the growth of strain-free GaAs/(Al, Ga)As QDs in locally etched pits [14]. Though these methods can be sufficient to enable the observation of entangled light from quantum dots [2,9,10], they are unsuitable for entangled light emission in the conventional fiber-transmission window around $1550 \mathrm{~nm}$, for which an alternative approach is required.

Here, we demonstrate that droplet epitaxy (DE) is a viable route to achieving QDs with small FSS, extending to conventional telecom wavelengths. In addition, we show the method is suitable for growth on standard (001) surfaces preferred for high-quality optical cavity growth. We investigate two material systems: InAs/GaAs and InAs/InP grown by two different growth techniques: molecular beam epitaxy (MBE) and metalorganic vapor-phase epitaxy (MOVPE) producing dots at 900 and $1550 \mathrm{~nm}$. The growth approach relies on the formation of metal droplets on the semiconductor substrate followed by crystallization in an arsenic environment. The density and size of the droplets can be independently controlled with deposition temperature and metal volume, respectively. Moreover, since the formation of DE QDs is not strain driven, the shape of the QD is less elongated, and their FSS remains small. 

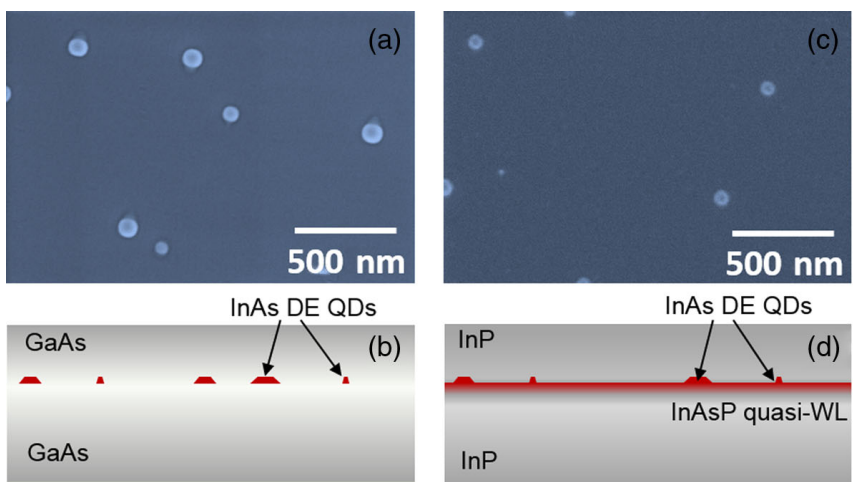

For fiber-optic applications, such as quantum communications, it is important that the dots be emitted in the telecom Fbands. In this respect, InAs/InP QDs are of particular interest, as the reduction in strain compared to InAs/GaAs QDs lengthens the emission wavelength to the conventional telecom band around $1550 \mathrm{~nm}$. InAs QDs on InP can be grown epitaxially by MOVPE or MBE, however, achieving low density is far more challenging than on GaAs substrates, without etching of nanomesas $[15,16]$. In order to reduce the dot densities and shift the emission wavelength, GaAs interlayers [17] and double-capping methods [18] have been proposed. Another problem is the formation of single quantum dashes rather than dots directly on InP $[15,19]$. Employing the droplet-epitaxy growth technique on InP enabled us to meet the low-density criteria at required emission wavelengths necessary for single-dot spectroscopy, and also to address the formation of dashes.

\section{EPITAXY}

In the Stranski-Krastanow (SK) growth mode of InAs quantum dots, arsenic and indium fluxes are supplied to the substrate at the same time. Initially, growth is two dimensional, and a highly strained InAs wetting layer is formed. The strain, caused by lattice mismatch between the InAs and the GaAs/InP substrate, increases with the thickness of the wetting layer. Once the wetting layer reaches a critical thickness, typically 1-2 monolayers, it becomes energetically favorable to form QDs, where the local strain can be accommodated. The structural symmetry of SK QDs is therefore intrinsically linked to the strain symmetry of the crystal substrate, in addition to subsequent preferential crystal growth in the [110] direction, and underlying rotational asymmetry of the atomic surface.

Droplet epitaxy, on the other hand, does not rely on strain during QD formation. Indium and arsenic fluxes are supplied separately at a lower temperature compared to SK QDs [see Fig. 1(e) for an illustration]. The QDs are formed first through the creation of liquid-indium droplets on the substrate, followed by a reaction with arsenic to form crystalline structures. The formation of a wetting layer is therefore avoided. Droplet epitaxy has been employed where SK QD growth is infeasible, including lattice-matched systems such

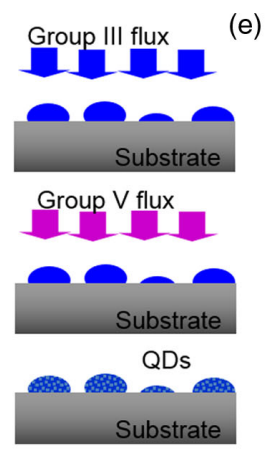

FIG. 1. Illustration of droplet formation. Scanning electron micrographs of uncrystallized indium droplets deposited on GaAs (001) (a) and InP (001) surface (c). (b) and (d) are cross-section illustrations of the DE QDs grown in GaAs and InP, respectively. The droplet-epitaxy growth scheme is illustrated in (e): a substrate is deposited with group-III material followed by crystallization with a group- $V$ flux leading to the formation of quantum dots.

as GaAs/AlAs, and especially on (111) crystal surfaces where the formation of high-quality two-dimensional layers is difficult. DE QDs grown on (001) surfaces with lattice mismatch have so far been limited, even though it has been suggested that the liquid nature of the droplet produces dislocation-free coherent nanocrystals [20].

MOVPE-grown DE QDs on InP are formed in a lowpressure reactor by the deposition of $\sim 2$ ML (mono layers) of In droplets on the InP surface via the pyrolysis of trimethylindium at $400{ }^{\circ} \mathrm{C}$ while withholding the supply of arsine to the growth chamber. The chamber pressure is 150 Torr during this process. The deposition rate of In is equivalent to a growth rate of InAs of $0.04 \mathrm{~nm} / \mathrm{s}$. The QD crystallization process under arsine overpressure starts at $400{ }^{\circ} \mathrm{C}$ and carries on until the substrate reaches $500^{\circ} \mathrm{C}$. Next, the dots are capped with $30 \mathrm{~nm}$ of $\mathrm{InP}$ followed by more InP at $640^{\circ} \mathrm{C}$. Trimethylindium, trimethylgallium, trimethylaluminium, phosphine, and arsine are used as precursors. The carrier gas is hydrogen. For surface dot characterization the growth process is stopped after In deposition [Fig. 1(b)] or after droplet crystallization [Fig. 2(b)].

SK growth by MOVPE on InP is performed at a temperature of $500^{\circ} \mathrm{C}$. A low growth rate of $0.05 \mathrm{~nm} / \mathrm{s}$ is employed while restricting the nominal thickness of InAs to $\sim 2 \mathrm{ML}$, which promoted a low-density QD growth. The dots are capped with $30 \mathrm{~nm}$ of $\mathrm{InP}$ at $500^{\circ} \mathrm{C}$ followed by InP at $640^{\circ} \mathrm{C}$.

Short-wavelength DE QDs are grown by MBE on GaAs (001) substrates. 1.4 ML of In is deposited at $390^{\circ} \mathrm{C}$ on a Ga-terminated GaAs buffer. The crystallization process involves supplying $\mathrm{As}_{4}$ to the growth chamber and ramping the temperature to $500^{\circ} \mathrm{C}$ followed by capping the dots with GaAs. Figure 1(a) shows In droplets deposited at $390^{\circ} \mathrm{C}$. The substrate is immediately cooled down for surface characterization.

In all three cases of dot formation described above, a dot density less than $10^{9} \mathrm{~cm}^{-2}$ is achieved.

The telecom device comprises a 20-pair DBR mirror, each pair consisting of $112 \mathrm{~nm}$ of $\left(\mathrm{Al}_{0.30} \mathrm{Ga}_{0.70}\right)_{0.48} \mathrm{In}_{0.52} \mathrm{As}$ and $123 \mathrm{~nm}$ of InP followed by $5 \lambda / 4 \mathrm{InP}$ and dots capped with $6 \lambda / 4 \mathrm{InP}$. The short-wavelength device consists of a 12-pair bottom DBR mirror, each pair consisting of $65 \mathrm{~nm}$ 

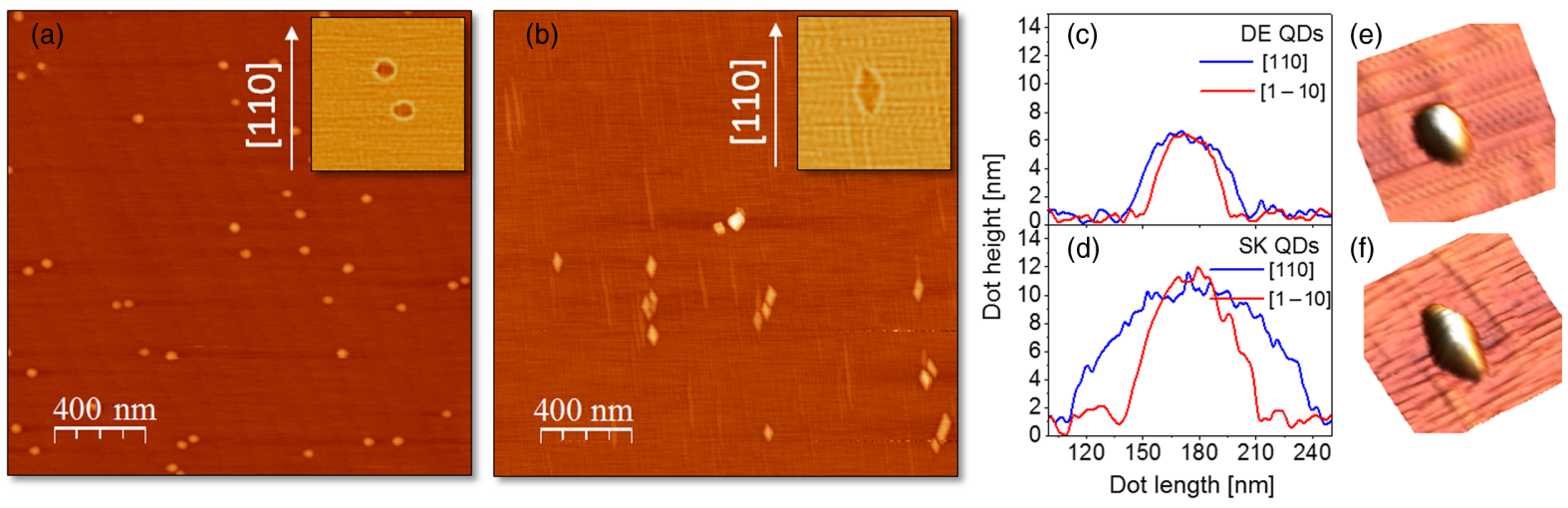

FIG. 2 Comparison of uncapped SK and DE dots grown on InP. Atomic force microscope (AFM) images of metalorganic vapor-phase epitaxy (MOVPE) crystallized DE QDs on InP (001) surface (a) and SK QDs on InP (001) surface (b). The scan area in both cases is $2 \times 2 \mu \mathrm{m}^{2}$. The insets are enlarged images of individual dots. Line scans over a typical DE QD and SK QD on InP are shown on (c) and (d), respectively. (e) and (f) are 3D representations of AFM scans of DE QD and SK QD on InP, respectively.

of GaAs and $77 \mathrm{~nm}$ of AlAs followed by a $2 \lambda$ GaAs cavity comprising dots in the middle. The cavity is finished off with two repeats of the top mirror.

Examples of indium droplets on GaAs and InP surfaces are shown in Figs. 1(a) and 1(c). The growth technique is presented in Fig. 1(e). Independent of the surface they are grown on, indium droplets are round and symmetric. The droplets' diameter has a mean of $130 \mathrm{~nm}$ and varies with a standard deviation of $38 \mathrm{~nm}$ in the case of the GaAs substrate, while the dots on the InP surface tend to be smaller, and of a diameter of $70 \mathrm{~nm}$, with a standard deviation of $10 \mathrm{~nm}$. The droplet density is $2-4 \times 10^{8} \mathrm{~cm}^{-2}$ in both cases. As the droplet-epitaxy technique is not strain driven, DE QDs formed on GaAs do not form a wetting layer typical for SK QDs. However, since droplets are deposited on InP and crystallized with arsenic, a "quasiwetting" layer can be formed as a result of the group- $\mathrm{V}$ element intermixing [21,22]. This is indicated in Figs. 1(b) and 1(d).

\section{SURFACE DOTS CHARACTERISATION}

A comparison of uncapped SK and DE quantum dots grown on $\mathrm{InP}$ (001) is characterized by AFM analysis (Fig. 2). A number of dot AFM images are fitted with ellipses for statistical analysis of the dot aspect ratio $\left(R_{x} / R_{y}\right)$ and the angle of elongation. The crystallization process of indium droplets into InAs QDs does not influence the circular symmetry of the dots significantly as illustrated by the MOVPE grown DE QDs crystallized on the surface of InP shown in Fig. 2(a). The DE QDs remain symmetric with a mean in-plane aspect ratio of 0.91 , and a standard deviation of 0.16 . An atomic force microscope (AFM) image of similar SK dots grown by MOVPE on InP is shown in Fig. 2(b). Here, the dots have a rhombic base, elongated towards [110]. The mean value of their aspect ratio is 0.53 and there is a standard deviation of 0.13 . Moreover, pronounced dashes can also be observed. Line scans over a typical DE and SK QDs are presented in
Figs. 2(c) and 2(d) showing the difference in the dot profile along orthogonal crystallographic directions, which is pronounced for SK QDs. This is also observed in corresponding AFM images of individual DE and SK QDs shown in Figs. 2(e) and 2(f), visualizing the 72\% improvement in the aspect ratio of the DE QDs compared to SK QDs emitting at the same wavelength. As shown in Fig. 2, DE QDs on InP are smaller than equivalent SK QDs. The smaller size is expected to increase the strength of the Coulomb interaction and FSS, which on the other hand is ruling out the size difference as a cause of reduced FSS.

One of the chief advantages of the (001) surface is the well-established growth of high-quality optical microcavities. Such optical structures are used to greatly improve the collection efficiency of light emitted by QDs, and are widely utilized in single QD optical experiments.

\section{OPTICAL CHARACTERISATION}

Low-temperature microphotoluminescence $(\mu \mathrm{PL})$ measurements are performed with a 785-nm CW excitation laser and the samples are mounted in a liquid-heliumcooled cryostat. The emission is coupled into a single-mode fiber to isolate the signal from individual quantum dots. Sending the fiber-coupled light to a spectrometer equipped with a silicon CCD camera or (In, Ga)As photodiode array, we are able to perform spectrally resolved measurements.

Figure 3(a) shows a wide-range optical spectrum taken on an optical cavity with a DE QDs ensemble at $20 \mathrm{~K}$. The cavity mode is fitted with a red line with its central wavelength of $1551 \pm 0.35 \mathrm{~nm}$ and a FWHM of $30 \mathrm{~nm}$. The inset in Fig. 3(a) shows individual spectral lines from a single dot in the same sample. The room-temperature reflectance spectrum is shown in Fig. 3(b). Here, the cavity peak is centered at $1575 \mathrm{~nm}$, accounting for $\sim 20 \mathrm{~nm}$ blueshift related to temperature. The spectral width of the emission lines are typically limited by the spectrometer resolution of $\sim 35 \mu \mathrm{eV}$. 


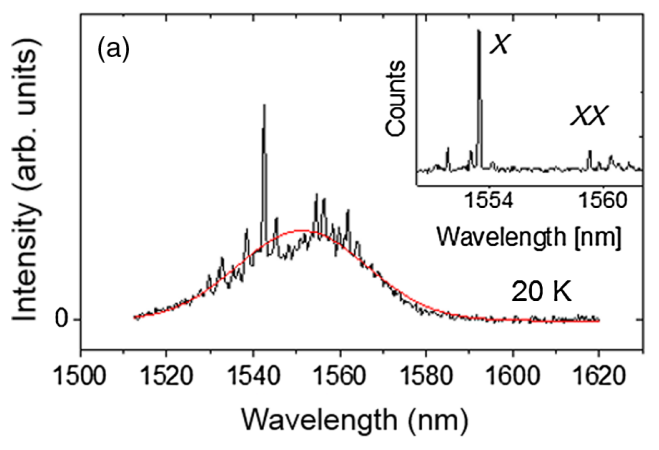

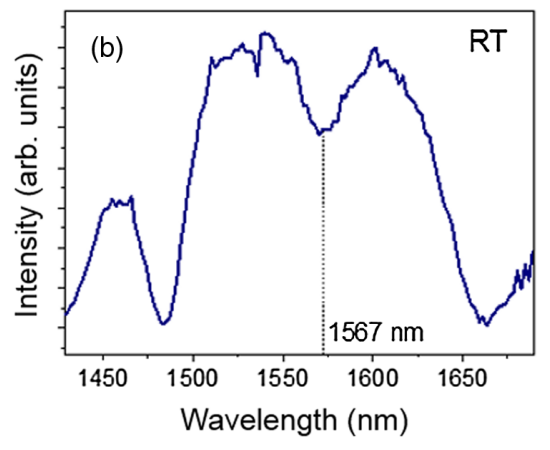

FIG. 3 (a) Spectrum taken on an optical cavity showing an ensemble of DE QDs at $20 \mathrm{~K}$. The cavity mode is present at the central wavelength of $1551 \pm 0.35 \mathrm{~nm}$ and $\mathrm{FWHM}=30 \mathrm{~nm}$. The inset shows a spectrum taken on an individual dot with a fine grating of the spectrometer. (b) RT reflectance spectrum taken on the same optical cavity showing the cavity peak centered at $1567 \mathrm{~nm}$.
FSS measurements are performed by sending the photoluminescence light from a single dot through a rotatable wave plate and fixed linear polarizer, and measuring the resulting shift in energy at the spectrometer. We employ two variations of this technique. The first uses a half-wave plate (HWP), which is the established technique to measure FSS of quantum dots [6,7]. The second method uses a quarter-wave plate (QWP), which we show can extract information on the FSS, the orientation of the quantum dot's dipole moment with respect to the lab frame, and the birefringence accumulated in the optical fiber. This differs from the traditional half-wave-plate technique, which provides just the FSS and dipole orientation, limiting it to free-space applications.
In our experiments, we employ the QWP method for InAs/InP DE QDs, on light emerging from single-mode fiber, and the HWP method for InAs/GaAs DE QDs, in free space before coupling into fiber. Where the FSS is below the resolution of the spectrometer, we could observe shifts by performing fits to the line shape, providing an accuracy of $\sim 2 \mu \mathrm{eV}$.

\section{RESULTS}

Figure 4 shows the spectra corresponding to a small number of QDs emitting in the cavity mode for (a) SK QDs on InP emitting around $1550 \mathrm{~nm}$, (b) DE QDs on InP emitting around $1550 \mathrm{~nm}$, and (c) DE QDs on GaAs emitting around $900 \mathrm{~nm}$. The excitonic transitions are
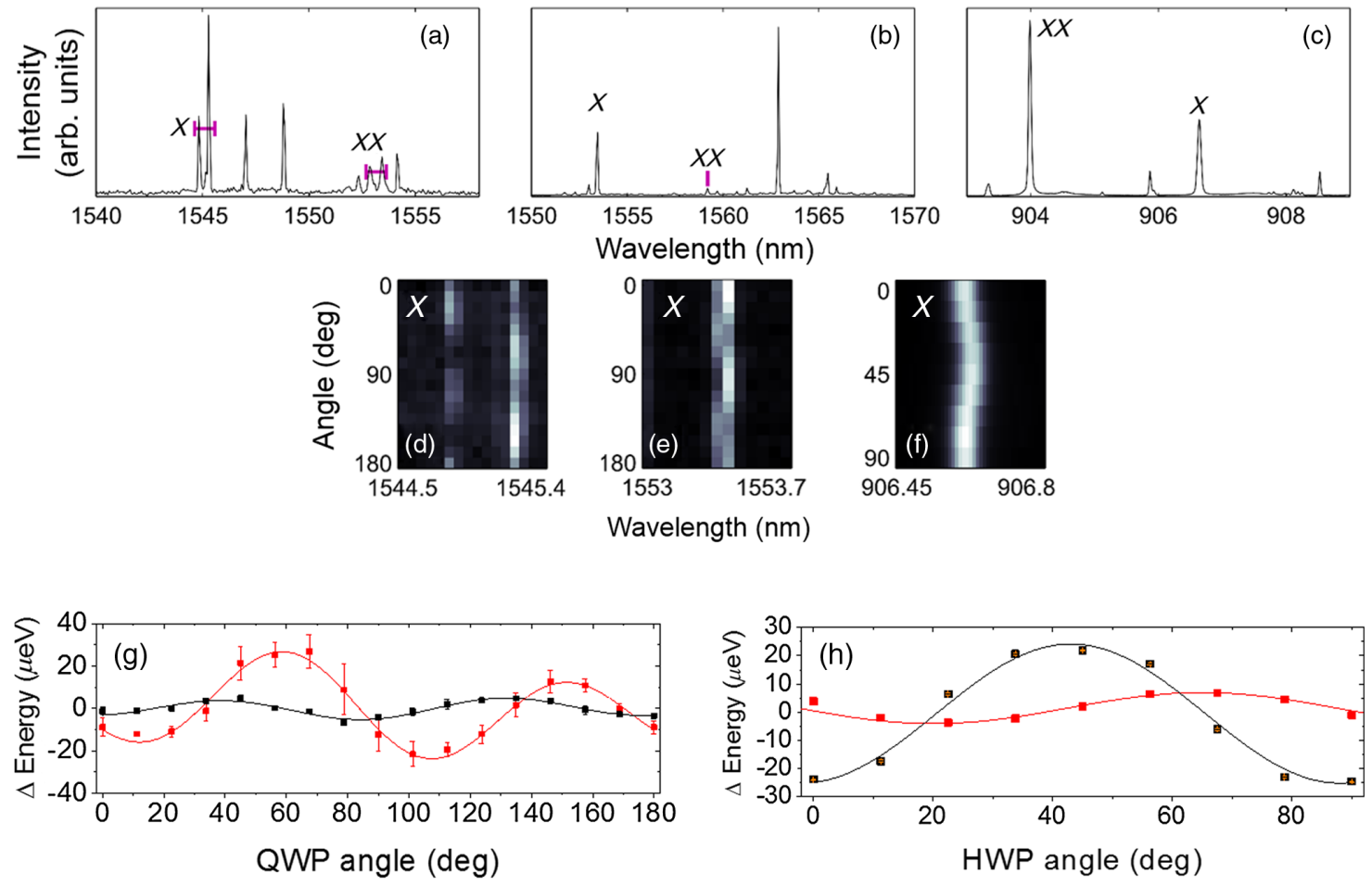

FIG. 4 (a)-(c) Spectra of individual InAs quantum dots for (a) SK QDs on InP emitting at $1550 \mathrm{~nm}$, (b) DE QDs on InP emitting at $\sim 1550 \mathrm{~nm}$, and (c) DE QDs on GaAs emitting at $\sim 900 \mathrm{~nm}$. $X$ and $X X$ transitions are identified. (d)-(f) Corresponding polarized $X$ emission spectra as a function of the angle of a quarter-wave plate (d),(e) or half-wave plate (f). (g),(h) Example FSS measurements of (g) DE QDs on InP emitting at $\sim 1550 \mathrm{~nm}$, and (h) DE QDs on GaAs emitting at $900 \mathrm{~nm}$. Each panel comprises two example measurements for $X$ lines with small and large FSS. For details, see text. 


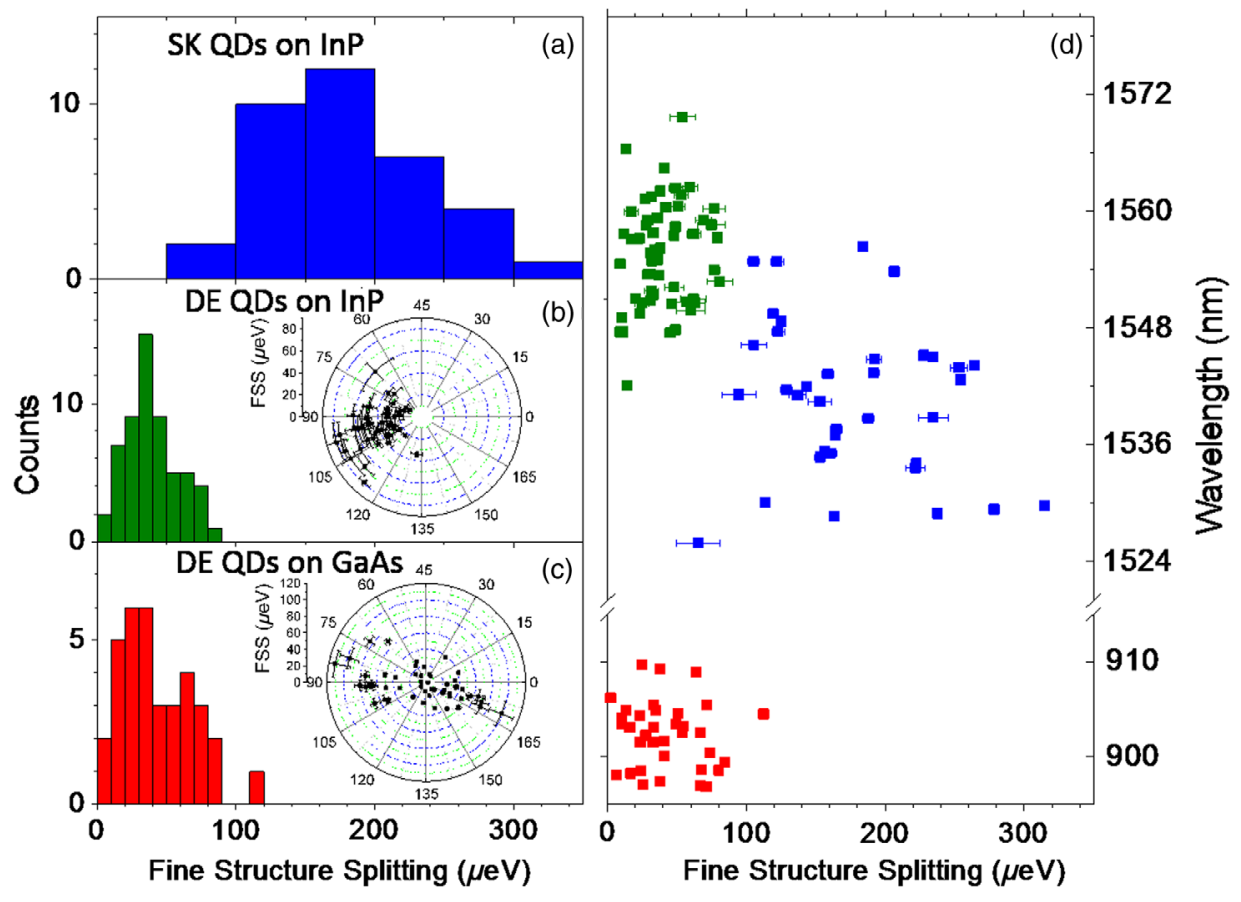

FIG. 5. Exciton fine-structure splitting of quantum dots emitting at around 900 and $1550 \mathrm{~nm}$ (telecom $C$ band). (a) FSS histogram for SK QDs grown on InP emitting at $1550 \mathrm{~nm}$. (b) FSS histogram for DE QDs grown on InP emitting around $1550 \mathrm{~nm}$. (c) FSS histogram for DE QDs grown on GaAs emitting around $900 \mathrm{~nm}$. (d) Corresponding FSS dependence on the emission wavelength measured for DE (green) and SK (blue) QDs grown on InP, and DE QDs grown on GaAs (red). The insets in (b) and (c) show polar plots for FSS values measured for DE QDs grown on InP and GaAs, respectively. identified by power dependence and FSS measurements. Typical binding energies $X X$ states in DE QDs are $\sim 3 \mathrm{meV}$, with both bound and antibound configurations observed. The plots (d)-(f) below each panel (a)-(c) indicate the spectral shifts on the exciton $(X)$ line in each spectrum when filtering the emission for different polarizations, as a function of the wave-plate angle. The FSS value for the $X$ line in Fig. 4(a) is $235 \pm 3 \mu \mathrm{eV}$, which is typical for SK dots at $\sim 1550-\mathrm{nm}$ wavelengths. FSS measured on DE QDs is significantly smaller: $29 \pm$ $1 \mu \mathrm{eV}$ around $1550 \mathrm{~nm}$ in Fig. 4(b) and $31 \pm 1 \mu \mathrm{eV}$ for DE QDs at around $900 \mathrm{~nm}$ in Fig. 4(c). The range of QD emission wavelengths observed is limited by the transmission band of the cavity mode. Thus, QD emission over a wide range within the conventional and neighboring telecom bands may be achieved by a modification of the optical cavity alone.

Example FSS measurements are shown in Figs. 4(g) and 4(h) for DE QDs on InP and DE QDs on GaAs, respectively. Energy shifts as a function of the wave-plate angle are well resolved, and fit well with the expected behavior (solid lines). A more complex dependence is observed for the QWP method, due to the presence of richer information compared to the HWP method. For the examples shown here, we measure 17 and $79 \mu \mathrm{eV}$ for the FSS of DE QDs on InP, and 10 and $49 \mu \mathrm{eV}$ for the FSS of DE QDs on GaAs.

Figure 5 summarizes the statistics of FSS taken on both $\mathrm{DE}$ and SK QDs grown on InP and GaAs. The distribution of FSS for SK QDs on InP emitting around $1550 \mathrm{~nm}$ is shown in Fig. 5(a). The FSS is found to be very large, with a measured mean value of $176 \pm 9 \mu \mathrm{eV}$. This is attributed to highly elongated quantum dots [23], confirmed by the mean aspect ratio of 0.53 measured by AFM for uncapped SK QDs on InP. For such large FSS, a significant reduction through annealing [24] or the use of external magnetic [25], electric [26,27], or strain fields [28,29] is impractical.

The difference between SK dots and DE QDs grown on InP is substantial. The FSS measured for DE QDs is more than $4 \times$ smaller and has a mean value of $42 \pm 7 \mu \mathrm{eV}$ [see Fig. 5(b)], attributed to the more symmetric base aspect ratio of 0.91 . Also, the distribution of FSS is significantly smaller for droplets compared to SK dots, with a standard deviation of $17 \mu \mathrm{eV}$ compared to $58.8 \mu \mathrm{eV}$ for SK dots.

Similar statistical analysis is carried out for DE QDs dots grown on GaAs emitting around $900 \mathrm{~nm}$ [Fig. 5(c)]. The FSS distribution is remarkably similar to that of Fig. 5(b) for DE QDs grown on InP. The FSS distribution has a mean $42 \pm 4 \mu \mathrm{eV}$, with a standard deviation $30.5 \mu \mathrm{eV}$.

The polar plots in Figs. 5(b) and 5(c) show the QD dipole orientation measured for DE QDs grown on InP and GaAs, respectively. DE QDs on InP tend to have dipoles aligned along one privileged direction. DE QDs on GaAs tend to have a more random dipole distribution, particularly for FSS less than $40 \mu \mathrm{eV}$. This may be associated with the lack of a wetting layer and with the fact that larger dots have smaller base aspect ratios.

\section{CONCLUSIONS}

Previous reports have shown a correlation between the emission wavelength and the FSS of the quantum dot for both SK dots [7] and DE dots [8]. However, for the DE QDs 
studied here, we observe no obvious correlation, as shown in Fig. 4(d). This has a particular consequence for DE QDs emitting around $900 \mathrm{~nm}$, as it lifts the restriction to operate at the wavelength corresponding to minimum FSS, typically $885 \mathrm{~nm}$. We also observe no dependence on the FSS for SK QDs with an emission wavelength at around $1550 \mathrm{~nm}$, which suggests that the QD shape can vary independently from the size for both growth techniques.

The realization of quantum-dot-based entangled light sources for the conventional telecom band requires quantum dots emitting around $1550 \mathrm{~nm}$, with small FSS, and embedded in an optical microcavity. Our results show such quantum dots can be produced using a droplet epitaxy of InAs QDs on (001) InP substrates. The reduction in FSS compared to an SK QD sample grown at a similar wavelength is a factor of 4 smaller, and critically the fraction of QDs with less than $40 \mu \mathrm{eV}$ splitting (the mean FSS value for $\mathrm{DE}$ QDs) is increased from $0 \%$ to $53 \%$. The smallest FSS measured for a DE QD on InP here is $12 \pm 2 \mu \mathrm{eV}$.

In summary, the growth of InAs droplets on (001) surfaces provides a universal growth scheme, allowing QDs to be realized within optical microcavities, with a similar low FSS distribution, but on different semiconductor materials, and with emission wavelengths separated by $\sim 600 \mathrm{~nm}$. Our study suggests a possible route to produce low FSS QDs at any wavelength between 900 and $1550 \mathrm{~nm}$. Finally, we demonstrate that the technique can produce entanglement-ready quantum dots in the conventional telecom band around $\sim 1550 \mathrm{~nm}$, within an optical microcavity. This is an important step towards the realization of a QD-based entangled light emitter for quantum communications and quantum cryptography applications.

\section{ACKNOWLEDGMENTS}

The authors would like to acknowledge partial financial support of UK EPSRC and Innovate UK (Projects No. 102245 and No. EP/M013472/1). We also would like to thank Guillaume Bourmaud for writing the AFM analysis software code.

\section{APPENDIX: FINE-STRUCTURE SPLITTING MEASUREMENTS}

Suppose our quantum dots emit light in the state $\rho_{1}$, where the $H$ and $V$ polarizations are eigenstates of the Hamiltonian $\hat{H}$,

$$
\rho_{1}=\left(\frac{1+p}{2}\right)|H\rangle\left\langle H\left|+\left(\frac{1-p}{2}\right)\right| V\right\rangle\langle V|,
$$

$\hat{H} \rho_{1}=E_{H}\left(\frac{1+p}{2}\right)|H\rangle\left\langle H\left|+E_{V}\left(\frac{1-p}{2}\right)\right| V\right\rangle\langle V|$,

with energies $\mathrm{EH}$ and $E V$, respectively. In the case that degeneracy of these states is lifted, they will exhibit a finestructure splitting $s=E H-E V$, and the mean energy is given by $\varepsilon=(E H+E V) / 2$. The state may also have some polarization $p$.

After traveling through a series of optics and fibers, typically birefringent media, the state will have undergone a rotation $\theta$ and phase shift $\varphi$ in its polarization.

$$
\begin{gathered}
|H\rangle \rightarrow\left|B_{1}\right\rangle=\cos \frac{\theta}{2}|H\rangle+\sin \frac{\theta}{2} e^{i \phi}|V\rangle, \\
|V\rangle \rightarrow|B\rangle_{2}=\sin \frac{\theta}{2}|H\rangle-\cos \frac{\theta}{2} e^{i \phi}|V\rangle, \\
\rho_{1} \rightarrow \rho_{2}=\left(\frac{1+p}{2}\right)\left|B_{1}\right\rangle\left\langle B_{1}\left|+\left(\frac{1-p}{2}\right)\right| B_{2}\right\rangle\left\langle B_{2}\right| .
\end{gathered}
$$

Immediately before entering our spectrometer, the light is passed through a quarter-wave plate (QWP) at an angle $\chi$ to the transmission polarization, that we call $H$, of a subsequent linear polarizer. (In the case that our measurement $H$ polarization does not precisely correspond to one of the eigenbases from the first equation, we will see some constant offset in $\theta$.) This is equivalent to measuring our state against the measurement basis $|M\rangle$, given by

$$
\begin{aligned}
|M(\chi)\rangle= & \operatorname{QWP}(\chi)|H\rangle=\frac{1}{\sqrt{2}}(i+\cos 2 \chi)|H\rangle \\
& +\frac{1}{\sqrt{2}} \sin 2 \chi|V\rangle .
\end{aligned}
$$

The energy we observe at the spectrometer is

$$
\begin{aligned}
E(\chi) & =\frac{\left\langle M\left|\hat{H} \rho_{2}\right| M\right\rangle}{\left\langle M\left|\rho_{2}\right| M\right\rangle} \\
& =\varepsilon+\frac{s}{2}\left(\frac{\left(\alpha_{1}-\alpha_{2}\right)+p}{1+p\left(\alpha_{1}-\alpha_{2}\right)}\right),
\end{aligned}
$$

where $\alpha_{j}=\left|\left\langle M \mid B_{j}\right\rangle\right|^{2}$. We thereby get an expression for the deviation $\Delta E$ from the mean energy $\varepsilon$ as a function of $\chi$

$$
\begin{aligned}
\alpha_{1}-\alpha_{2}= & \frac{1}{2}[\cos \theta(1+\cos 4 \chi)+\sin \theta \sin 4 \chi \cos \phi \\
& -2 \sin \theta \sin 2 \chi \sin \phi],
\end{aligned}
$$


[1] O. Benson, C. Santori, M. Pelton, and Y. Yamamoto, Regulated and Entangled Photons from a Single Quantum Dot, Phys. Rev. Lett. 84, 2513 (2000).

[2] R. M. Stevenson, R. J. Young, P. Atkinson, K. Cooper, D. A. Ritchie, and A. J. Shields, A semiconductor source of triggered entangled photon pairs, Nature (London) 439, 179 (2006).

[3] C. L. Salter, R. M. Stevenson, I. Farrer, C. A. Nicoll, D. A. Ritchie, and A.J. Shields, An entangled-light-emitting diode, Nature (London) 465, 594 (2010).

[4] M. B. Ward, M. C. Dean, R. M. Stevenson, A. J. Bennett, D. Ellis, K. Cooper, I. Farrer, C. A. Nicoll, D. A. Ritchie, and A. J. Shields, Coherent dynamics of a telecomwavelength entangled photon source, Nat. Commun. 5, 3316 (2014).

[5] V. D. Kulakovskii, G. Bacher, R. Weigand, T. Kümmell, A. Forchel, E. Borovitskaya, K. Leonardi, and D. Hommel, Fine Structure of Biexciton Emission in Symmetric and Asymmetric CdSe/ZnSe Single Quantum Dots, Phys. Rev. Lett. 82, 1780 (1999).

[6] D. Gammon, E. S. Snow, B. V. Shanabrook, D. S. Katzer, and D. Park, Fine Structure Splitting in the Optical Spectra of Single GaAs Quantum Dots, Phys. Rev. Lett. 76, 3005 (1996).

[7] R. J. Young, R. M. Stevenson, A. J. Shields, P. Atkinson, K. Cooper, D. A. Ritchie, K. M. Groom, A. I. Tartakovskii, and M.S. Skolnick, Inversion of exciton level splitting in quantum dots, Phys. Rev. B 72, 113305 (2005).

[8] X. Liu, N. Ha, H. Nakajima, T. Mano, T. Kuroda, B. Urbaszek, H. Kumano, I. Suemune, Y. Sakuma, and K. Sakoda, Vanishing fine-structure splittings in telecommunication-wavelength quantum dots grown on (111)A surfaces by droplet epitaxy, Phys. Rev. B 90, 081301(R) (2014).

[9] T. Kuroda, T. Mano, N. Ha, H. Nakajima, H. Kumano, B. Urbaszek, M. Jo, M. Abbarchi, Y. Sakuma, K. Sakoda, I. Suemune, X. Marie, and T. Amand, Symmetric quantum dots as efficient sources of highly entangled photons. Violation of Bell's inequality without spectral and temporal filtering, Phys. Rev. B 88, 041306(R) (2013).

[10] G. Juska, V. Dimastrodonato, L. O. Mereni, A. Gocalinska, and E. Pelucchi, Towards quantum-dot arrays of entangled photon emitters, Nat. Photonics 7, 527 (2013).

[11] N. Ha, T. Mano, Y.-N. Wu, Y.-W. Ou, S.-J. Cheng, Y. Sakuma, K. Sakoda, and T. Kuroda, Wavelength extension beyond $1.5 \mu \mathrm{m}$ in symmetric InAs quantum dots grown on InP(111)A using droplet epitaxy, Appl. Phys. Express 9, 101201 (2016).

[12] N. Ha, T. Mano, T. Kuroda, K. Mitsuishi, A. Ohtake, A. Castellano, S. Sanguinetti, T. Noda, Y. Sakuma, and K. Sakoda, Droplet epitaxy growth of telecom InAs quantum dots on metamorphic InAlAs/GaAs(111)A, Jpn. J. Appl. Phys. 54, 04DH07 (2015).

[13] N. Ha, X. Liu, T. Mano, T. Kuroda, K. Mitsuishi, A. Castellano, S. Sanguinetti, T. Noda, Y. Sakuma, and K. Sakoda, Droplet epitaxial growth of highly symmetric quantum dots emitting at telecommunication wavelengths on $\operatorname{InP}(111) \mathrm{A}$, Appl. Phys. Lett. 104, 143106 (2014).
[14] Y. H. Huo, A. Rastelli, and O. G. Schmidt, Ultra-small excitonic fine structure splitting in highly symmetric quantum dots on GaAs (001) substrate, Appl. Phys. Lett. 102, 152105 (2013).

[15] Ł. Dusanowski, M. Syperek, P. Mrowiński, W. Rudno-Rudziński, J. Misiewicz, A. Somers, S. Höfling, M. Kamp, J. P. Reithmaier, and G. Sęk, Single photon emission at $1.55 \mu \mathrm{m}$ from charged, and neutral exciton confined in a single quantum dash, Appl. Phys. Lett. 105, 021909 (2014).

[16] T. Miyazawa, K. Takemoto, Y. Sakuma, S. Hirose, T. Usuki, N. Yokoyama, M. Takatsu, and Y. Arakawa, Single-photon generation in the $1.55-\mu \mathrm{m}$ optical-fiber band from an InAs/InP quantum dot, Jpn. J. Appl. Phys. 44, L620 (2005).

[17] M. D. Birowosuto, H. Sumikura, S. Matsuo, H. Taniyama, P. J. van Veldhoven, R. Nötzel, and M. Notomi, Fast Purcellenhanced single photon source in 1,550-nm telecom band from a resonant quantum dot-cavity coupling, Sci. Rep. 2, 321 (2012).

[18] K. Takemoto, Y. Sakuma, S. Hirose, T. Usuki, and N. Yokoyama, Observation of exciton transition in 1.3-1.55 $\mu \mathrm{m}$ band from single InAs/InP quantum dots in Mesa structure, Jpn. J. Appl. Phys. 43, L349 (2004).

[19] S. Anantathanasarn, R. Nötzel, P. J. van Veldhoven, T. J. Eijkemans, and J. H. Wolter, Wavelength-tunable $(1.55-\mu \mathrm{m}$ region) InAs quantum dots in InGaAsP/InP (100) grown by metal-organic vapor-phase epitaxy, J. Appl. Phys. 98, 013503 (2005).

[20] T. Mano, K. Watanabe, S. Tsukamoto, H. Fujioka, M. Oshima, and N. Koguchi, New self-organized growth method for InGaAs quantum dots on $\mathrm{GaAs}(001)$ using droplet epitaxy, Jpn. J. Appl. Phys. 38, L1009 (1999).

[21] N. Carlsson, T. Junno, L. Montelius, M.-E. Pistol, L. Samuelson, and W. Seifert, Growth of self-assembled InAs and $\operatorname{InAs} \mathrm{P}_{x} \mathrm{P}_{1-x}$ dots on InP by metalorganic vapour phase epitaxy, J. Cryst. Growth 191, 347 (1998).

[22] Ji-Myon Lee, Do Hyun Kim, Hoony Hong, Jong-Chun Woo, and Seong-Ju Park, Growth of InAs nanocrystals on GaAs(l 0 0 $)$ by droplet epitaxy, J. Cryst. Growth 212, 67 (2000).

[23] L. He, M. Gong, C.-F. Li, G.-C. Guo, and A. Zunger, Highly Reduced Fine-Structure Splitting in InAs/InP Quantum Dots Offering an Efficient On-Demand Entangled 1.55- $\mu \mathrm{m}$ Photon Emitter, Phys. Rev. Lett. 101, 157405 (2008).

[24] D. J. P. Ellis, R. M. Stevenson, R. J. Young, A. J. Shields, P. Atkinson, and D. A. Ritchie, Control of fine-structure splitting of individual InAs quantum dots by rapid thermal annealing, Appl. Phys. Lett. 90, 011907 (2007).

[25] R. M. Stevenson, R. J. Young, P. See, D. G. Gevaux, K. Cooper, P. Atkinson, I. Farrer, D. A. Ritchie, and A. J. Shields, Magnetic-field-induced reduction of the exciton polarization splitting in InAs quantum dots, Phys. Rev. B 73, 033306 (2006).

[26] A. J. Bennett, M. A. Pooley, R. M. Stevenson, M. B. Ward, R. B. Patel, A. B. de la Giroday, N. Sköld, I. Farrer, C. A. Nicoll, D. A. Ritchie, and A. J. Shields, Electric-fieldinduced coherent coupling of the exciton states in a single quantum dot, Nat. Phys. 6, 947 (2010). 
[27] K. Kowalik, O. Krebs, A. Lemaítre, B. Eble, A. Kudelski, P. Voisin, S. Seidl, and J.A. Gaj, Monitoring electrically driven cancellation of exciton fine structure in a semiconductor quantum dot by optical orientation, Appl. Phys. Lett. 91, 183104 (2007).

[28] J. Zhang, J. S. Wildmann, F. Ding, R. Trotta, Y. Huo, E. Zallo, D. Huber, A. Rastelli, and O. G. Schmidt, High yield and ultrafast sources of electrically triggered entangledphoton pairs based on strain-tunable quantum dots, Nat. Commun. 6, 10067 (2015).

[29] S. Seidl, M. Kroner, A. Högele, K. Karrai, R. J. Warburton, A. Badolato, and P. M. Petroff, Effect of uniaxial stress on excitons in a self-assembled quantum dot, Appl. Phys. Lett. 88, 203113 (2006). 